\title{
A Case Report on Ischaemic Cardiomyopathy with Severe Left Ventricular Dysfunction
}

\author{
Authors: \\ *Mohammed Misbah UI Haq, Mohammed Munaf Ur Razzak, Nazish \\ Ahmed \\ Department of Pharmacy Practice, Deccan School of Pharmacy, Hyderabad, India \\ *Correspondence to misbahjed@hotmail.com \\ Disclosure: $\quad$ The authors have declared no conflicts of interest. \\ Received: $\quad 05.11 .20$ \\ Accepted: $\quad 15.02 .21$ \\ Keywords: Ischaemic cardiomyopathy with severe left ventricular dysfunction, left ventricular \\ systolic dysfunction, severe ischaemic cardiomyopathy. \\ Citation: $\quad$ EMJ Cardiol. 2021;9[1]:84-91.
}

\begin{abstract}
Ischaemic cardiomyopathy is a condition that arises when heart muscle is weakened because of coronary artery disease or a heart attack. Left ventricular (LV) dysfunction occurs when the left ventricle is either defective or damaged, thus disrupting healthy function. Normal LV function can be perturbed because of several causes. Some cardiac defects such as valvular malformations or conditions block the passage of blood into the body. Effective and cost-effective treatment is available for such patients that can reduce both morbidity and mortality. Herein, the authors present the case of a 69-year-old male who was brought to the emergency department with a history of hypertension on medication. Later, the patient was transferred to the cardiology department. The patient was brought to the hospital after midnight and had bleeding gums, and experienced bleeding from the site of needle puncture. Earlier reports showed that the international normalised ratio was $>6.0$, and the 2D echocardiogram showed large LV blood clots, mild LV dysfunction, mild mitral regurgitation, and aortic valve stenosis. Finally, the patient was diagnosed with ischaemic cardiomyopathy associated with LV dysfunction. During discharge, the patient and patient's representative were counselled in layman's language about the conditions and prognosis of the disease, the use and adherence to medications, lifestyle modifications, and were advised to review back to the cardiologist.
\end{abstract}

\section{INTRODUCTION}

Cardiomyopathy is a group of diseases that affect the heart muscle. Early on, there may be few or no symptoms. As the disease worsens, shortness of breath, feeling tired, and swelling of the legs may occur, due to the onset of heart failure (HF). An irregular heartbeat and fainting may occur. ${ }^{1}$ Those affected are at an increased risk of sudden cardiac death. ${ }^{1}$

In 2015, cardiomyopathy and myocarditis affected 2.5 million people. ${ }^{2}$ Hypertrophic cardiomyopathy affected approximately one in 500 people while dilated cardiomyopathy affected one in 2,500;1,3 these conditions resulted in 354,000 deaths, increased from 294,000 in $19900^{2,4}$

Types of cardiomyopathy include hypertrophic cardiomyopathy, dilated cardiomyopathy, restrictive cardiomyopathy, arrhythmogenic right ventricular dysplasia, and Takotsubo cardiomyopathy (broken heart syndrome). 
Dilated cardiomyopathy is a condition in which the heart becomes enlarged and cannot pump blood effectively.' Symptoms vary from none to feeling tired, leg swelling, and shortness of breath. 'It may also result in chest pain or fainting. Complications can include HF, valvular heart disease, or an irregular heartbeat.'

Approximately one in 2,500 people are affected by cardiomyopathy. ${ }^{5}$ It occurs more frequently in males than females. ${ }^{1}$ Onset is most often in middle age. ${ }^{6}$ The 5 -year survival rate is approximately $50 \% .^{5}$ Although in many cases no cause is apparent, dilated cardiomyopathy is probably the result of damage to the myocardium produced by a variety of toxic, metabolic, or infectious agents. ${ }^{7}$ It can also occur in children and is the most common type of cardiomyopathy in this age group. ${ }^{5}$ It may be because of fibrous change to the myocardium from a previous myocardial infarction. Or it may be the late sequelae of acute viral myocarditis, such as with Coxsackie B virus and other enteroviruses, ${ }^{8}$ possibly mediated through an immunologic mechanism. ${ }^{7}$

Ischaemic cardiomyopathy (ICM) is the most common type of dilated cardiomyopathy. In ICM, the ability of the heart to pump blood is decreased because the heart's main pumping chamber, the left ventricle, is enlarged, dilated, and weak. This is caused by ischaemia: a lack of blood supply to the heart muscle caused by coronary artery disease and heart attacks.

Causes include genetics, alcohol, cocaine, certain toxins, complications of pregnancy, and certain infections. ${ }^{1,5}$ Coronary artery disease and high blood pressure may play a role, but are not the primary cause., ${ }^{6,7}$ In many cases, the cause remains unclear. ${ }^{1}$ It is a type of cardiomyopathy, a group of diseases that primarily affects the heart muscle. ${ }^{1}$ The diagnosis may be supported by an ECG, chest X-ray, or echocardiogram. ${ }^{5}$

Symptoms may include shortness of breath; swelling of the legs and feet (oedema); weight gain, cough, and congestion related to fluid retention; dizziness or lightheadedness; fatigue, inability to exercise, or carry out activities as usual; palpitations or fluttering in the chest because of arrhythmia; and fainting caused by irregular heart rhythms and abnormal responses of the blood vessels during exercise. Angina that occurs with exercise, physical activity, rest, or after meals is a less common symptom.'
Major risk factors of heart disease, such as family history, high blood pressure, smoking, diabetes, high blood cholesterol, and obesity can also place individuals at increased risk for cardiovascular disease and ICM.

In those with HF, treatment may include medications in the angiotensin-converting enzyme inhibitor, $\beta$-blocker, and diuretic families. ${ }^{5}$ A low-salt diet may also be helpful. ${ }^{6}$ In those with certain types of irregular heartbeat, blood thinners or an implantable cardioverter defibrillator (ICD) may be recommended. ${ }^{5}$ If other measures are not effective, a heart transplant may be an option in some. ${ }^{5}$

The progression of HF is associated with left ventricular (LV) remodelling, which manifests as gradual increases in LV end-diastolic and endsystolic volumes, wall thinning, and a change in chamber geometry to a more spherical, less elongated shape. This process is usually associated with a continuous decline in ejection fraction. The concept of cardiac remodelling was initially developed to describe changes that occur in the days and months following myocardial infarction. ${ }^{9}$

\section{CASE REPORT}

A 69-year-old male presented to a tertiary care hospital with a large LV clot and on warfarin $5 \mathrm{mg}$ oral anticoagulant, which had induced bleeding. The patient's chief complaints were of bleeding from the gums and bleeding at the site of needle puncture for 1 day. At presentation, patient reports showed an international normalised ratio (INR) >6.O. In addition, 2D echocardiography also detected abnormalities, including a large clot in the LV apex, mild LV dysfunction, mild mitral regurgitation (MR), and aortic valve stenosis. The patient had a history of hypertension and was on medication. His vitals on 4 consecutive days are shown in Table 1.

Personal medical history revealed that the patient was a non-alcoholic and non-smoker, appetite was normal, sleep was adequate, and bowel and bladder functions were regular. The patient did not have any other comorbidities and no significant family history was noted. 
Table 1: The patient's vitals on 4 consecutive days.

\begin{tabular}{|l|l|l|l|l|l|}
\hline Vitals & Day 1 & Day 2 & Day 3 & Day 4 & Units \\
\hline Blood pressure & $160 / 70$ & $130 / 90$ & $110 / 60$ & $130 / 60$ & $\mathrm{mmHg}$ \\
\hline Heart rate & 68 & 90 & 92 & 88 & Beats/min \\
\hline Respiratory rate & 40 & 20 & 22 & 23 & Breaths/min \\
\hline $\begin{array}{l}\text { Cardiovascular } \\
\text { system }\end{array}$ & S1S2+ & S1S2+ & S1S2+ & S1S2+ & N/A \\
\hline Per abdomen & Soft, non-tender & Soft, non-tender & Soft, non-tender & Soft, non-tender & N/A \\
\hline
\end{tabular}

N/A: not applicable.

Physical examination showed that the patient was conscious, co-operative, and responding properly. The patient was feeling weak, with a blood pressure of $160 / 70 \mathrm{mmHg}$ on medication.

On cardiac auscultation, there was a regular heartbeat with no murmur heard and a heart rate of 89 beats/min was recorded. Respiratory auscultation revealed symmetrical breath sounds and normal bronchial airway entry with a respiratory rate of 20 breaths/min. The patient's abdomen was soft, regular, and non-tender.

Initial laboratory investigation showed that prothrombin time (PT) and activated partial thromboplastin time were $>1 \mathrm{~min}$, and high-sensitivity troponin-I levels were $36 \mathrm{ng} / \mathrm{L}$. Furthermore, 2D colour Doppler echocardiography revealed notable features of the patient's heart muscle structure and function, such as dilated LV; regional wall motion abnormality; hypokinesia of lateral wall, apex, and anteroseptal wall; mild LV dysfunction; mild MR; mild tricuspid regurgitation (TR); moderate aortic regurgitation; mild pulmonary arterial hypertension; and no pulmonary embolism, clot, or vegetations. Electrocardiography revealed sinus bradycardia with first-degree atrioventricular block, left ventricular hypertrophy with repolarisation abnormality, and an abnormal ECG. Other lab investigations such as complete blood picture, lipid profile, liver function test, and serum electrolytes were normal.
The patient was given an intravenous injection of tranexamic acid ( $40 \mathrm{mg}$ ), an injection of pantoprazole (40 $\mathrm{mg}$ once daily [qd]), an injection of ondansetron (4 mg twice daily), and an intramuscular qd injection of vitamin $\mathrm{K}$. Furosemide plus amiloride tablets ( $40 \mathrm{mg}$ qd), digoxin tablets ( $0.25 \mathrm{mg} \mathrm{qd}$ ), telmisartan plus hydrochlorothiazide tablets $(40 \mathrm{mg} / 12.5 \mathrm{mg}$ qd), metoprolol succinate tablets ( $25 \mathrm{mg} \mathrm{qd}$ ), and rosuvastatin tablets (10 $\mathrm{mg}$ nightly) were also administered.

PT and INR were addressed on the day of admission. On Day 2, the patient had no further episodes of bleeding and was advised to continue the same treatment. The patient was also advised to have PT and INR tested daily. On Day 3, the patient had no fresh complaints; he was conscious, coherent, and co-operative, and INR was found to be 3.9. The patient was discharged on the fifth day with the following medications: pantoprazole $40 \mathrm{mg}$ qd; furosemide plus amiloride $40 \mathrm{mg} / 5 \mathrm{mg}$ qd; digoxin 0.25 $\mathrm{mg}$ qd; telmisartan plus hydrochlorothiazide 40 $\mathrm{mg} / 12.5 \mathrm{mg}$ qd; rosuvastatin $10 \mathrm{mg}$ nightly; and metoprolol succinate $25 \mathrm{mg}$ qd.

\section{DISCUSSION}

The INR is a calculation based on results of PT and is used to monitor individuals who are being treated with the blood-thinning 
medication (anticoagulant) warfarin. The PT and INR are used to monitor the effectiveness of the anticoagulant warfarin.

HF due to ischaemic or dilated cardiomyopathy is associated with a significant increase in the risk of thromboembolism. Unless there are contraindications, anticoagulant treatment is mandatory for patients with HF and atrial fibrillation. HF is an independent risk factor for over-anticoagulation. Therefore, patients with HF should be closely monitored to prevent potential bleeding complications; anticoagulants have to be adjusted accordingly. ${ }^{10}$

Elevated pulmonary arterial pressure has been established as a predictor of death in patients with HF with both ischaemic and non-ischaemic cardiomyopathy. ${ }^{11}$

MR can be found in a sizeable percentage of patients with chronic congestive HF and systolic LV dysfunction despite a structurally normal valve. This functional or secondary regurgitation results from a dysbalance between closing and opening forces on the mitral leaflets because of reduced LV contractility, geometric distortion of the sub-valvular apparatus, and global dilatation of the left ventricle and the mitral annulus. MR in LV dysfunction has a negative impact on both symptoms and prognosis. MR is common and independently predicts mortality in patients with LV systolic dysfunction. Its management remains challenging because of the complexity and variety of potential mechanisms implicated. ${ }^{12,13}$

TR aetiologies are currently divided into primary and secondary TR. Intrinsic abnormalities of the tricuspid valve leading to significant TR (primary) are rare and are seen in approximately $8-10 \%$ of patients with severe TR. ${ }^{14,15}$ In contrast, secondary TR is the most frequent form of TR requiring surgical intervention. Secondary TR occurs mainly from tricuspid annular dilatation and increased tricuspid leaflet tethering because of right ventricular enlargement, which is often secondary to left HF from myocardial or valvular causes. ${ }^{16,17}$

Aortic stenosis (AS) is one of the most common and serious valve disease problems. AS is a narrowing of the aortic valve opening, which restricts the blood flow from the left ventricle to the aorta and may also affect the pressure in the left atrium. The correlation between the severity of AS and onset of symptoms is poor and depends largely on the hypertrophic response of the left ventricle to the pressure overload. ${ }^{18}$

LV hypertrophy is a compensatory mechanism to restore wall stress and maintain cardiac output under increasing pressure afterload caused by the stenotic valve. However, progressive cardiomyocyte death and consequent fibrosis that accompanies LV hypertrophy may lead to the development of LV dysfunction and HF symptoms. In addition, the frequent association of AS and MR can also complicate the aetiological contributors to HF. However, the coexistence of severe AS, reduced LVEF, and $\mathrm{HF}$ is complex and poses diagnostic and clinical decision-making dilemmas. ${ }^{18,19}$

Mitral annulus calcification (MAC) is a chronic, degenerative process of the fibrous support of the mitral valve. ${ }^{20,21}$ MAC is usually visualised on echocardiography as an echo-dense, shelf-like structure with an irregular, lumpy appearance involving the mitral valve annulus, with associated acoustic shadowing. ${ }^{22}$ MAC generally has little or no impact on LV inflow haemodynamics or mitral valve function. ${ }^{23}$ There are limited data suggesting that MAC may exacerbate MR. Patients with MAC have a higher prevalence of atrioventricular block, bundle branch block, intraventricular conduction delay, and atrial fibrillation. ${ }^{24}$

Among patients with HF, first-degree atrioventricular block is present in anywhere between 15\% and 51\%. Data from cardiac resynchronisation therapy studies have shown that first-degree atrioventricular block is associated with an increased risk of mortality and HF hospitalisation. ${ }^{25}$

Troponin $\mathrm{T}(\mathrm{TnT})$ is the best laboratory parameter in the diagnosis of myocardial injury. Because the cytoplasm of cardiomyocytes contains a small amount of free TnT, even small damage to the cell membrane causes their release and the possibility of detection in the blood sample under investigation. Therefore, TnT detected in plasma is a highly specific marker of myocardial injury. ${ }^{26,27}$ 


\section{DRUG-DRUG INTERACTIONS}

\section{Major Interactions}

\section{Digoxin and hydrochlorothiazide}

Onset is delayed and documentation is excellent. Concurrent use of digitalis glycosides and thiazide diuretics can result in digitalis toxicity (nausea, vomiting, and arrhythmias). It is hypothesised that this is because diureticinduced hypokalaemia and hypomagnesaemia enhance $\mathrm{Na}+\mathrm{K}+-\mathrm{ATPase}$ inhibition by cardiac glycosides.

Patients given diuretics with digitalis should be told to add rich sources of potassium to their diet or they should be given potassium supplements, even though their serum potassium level is normal. The use of potassium-sparing diuretics in combination with potassium-depleting diuretics is also a rational approach. Patients may want to include some extra potassium in their diet.

\section{Digoxin and telmisartan}

Onset is not specified and documentation is excellent. Concurrent use of digoxin and telmisartan may result in an increased risk of digoxin toxicity (nausea, vomiting, and arrhythmias). The probable mechanism is unknown.

Co-administration of digoxin and telmisartan may increase digoxin plasma concentrations to $50 \% .^{28,29}$

Measure digoxin concentrations prior to initiation of concurrent use. Reduce the digoxin dose (by approximately $15-30 \%$ for oral) or modify the dosing frequency. Continue monitoring digoxin plasma concentration. ${ }^{28}$

\section{Moderate Interactions}

\section{Amiloride hydrochloride and digoxin}

Onset is delayed and documentation is fair. Concurrent use of digoxin and amiloride may result in decreased digoxin effectiveness. The probable mechanism is unknown.

Clinical management should focus on monitoring patients for reduced therapeutic effect of digoxin.

\section{Amiloride hydrochloride and telmisartan}

Onset is not specified and documentation is fair. Concurrent use of telmisartan and potassiumsparing diuretics may result in an increased risk of hyperkalaemia. The probable mechanism is additive hyperkalaemia.

Hyperkalaemia may occur with concomitant use of potassium-sparing diuretics and telmisartan, with an increased risk of hyperkalaemia in patients with advanced renal impairment, HF, and those receiving renal replacement therapy, potassium supplements, salt substitutes, or other drugs that may increase potassium levels. Periodic electrolyte monitoring should be considered to detect possible electrolyte imbalances. ${ }^{29}$

\section{Digoxin and furosemide}

Onset is delayed and documentation is fair. Concurrent use of digoxin and loop diuretics may result in increased risk of digoxin toxicity (nausea, vomiting, and cardiac arrhythmias). This is because potassium and magnesium loss may enhance the effect of digoxin.

Frequent monitoring of potassium and possibly magnesium with appropriate replacement is recommended. Clinicians should educate patients about the importance of maintaining an adequate intake of dietary potassium and potassium supplements.

\section{Digoxin and metoprolol succinate}

Onset is not specified and documentation is good. Concurrent use of $\beta$-adrenergic blockers and digitalis glycosides may result in increased risk of bradycardia and possible digitalis glycoside toxicity. This is because of the additive effects on atrioventricular node conduction.

Both digitalis glycosides and $\beta$-blockers slow atrioventricular conduction and decrease heart rate, and concurrent use can increase the risk of bradycardia. If these drugs are co-administered, monitor heart rate and $\mathrm{PR}$ interval ${ }^{30}$ and use with caution. 


\section{DRUG-DISEASE INTERACTIONS}

\section{Severe Potential Hazard}

\section{Ondansetron and QT-interval prolongation}

ECG changes including QT interval prolongation have been observed in patients receiving ondansetron. Additionally, there have been some post-marketing reports of Torsade de Pointes cases. The use of ondansetron should be avoided in patients with congenital long QT syndrome. ECG monitoring is recommended in patients with electrolyte abnormalities such as hypokalaemia or hypomagnesaemia; patients with congestive HF or bradyarrhythmia; and patients taking other medicines that could lead to QT prolongation.

\section{Digoxin and preserved left ventricular ejection}

Patients with HF associated with preserved LV systolic function, such as in restrictive cardiomyopathy, constrictive pericarditis, amyloid heart disease, and acute cor pulmonale may be particularly susceptible to the toxicity of digoxin. Therapy with digoxin should be considered and administered cautiously in such patients. In hypertrophic cardiomyopathy (idiopathic hypertrophic subaortic stenosis), the inotropic effects of digoxin may worsen the outflow obstruction.

\section{TREATMENT OPTIONS}

\section{Medical Therapy}

Drug therapy can slow down progression and, in some cases, improve the heart condition. Standard therapy may include salt restriction, angiotensin-converting enzyme inhibitors, diuretics, and $\beta$-blockers. ${ }^{31}$ Anticoagulants may also be used for antithrombotic therapy. There is some evidence for the benefits of coenzyme Q10 in treating HF. ${ }^{32-34}$

\section{Electrical Treatment}

Artificial pacemakers may be used in patients with intraventricular conduction delay and ICD in those at risk of arrhythmia. These forms of treatment have been shown to prevent sudden cardiac death, improve symptoms, and reduce hospitalisation in patients with systolic HF. ${ }^{35}$

\section{Surgical Treatment}

In patients with advanced disease who are refractory to medical therapy, heart transplantation may be considered. For these individuals, 1-year survival approaches 90\% and over $50 \%$ survive for more than 20 years. ${ }^{35}$

\section{CONCLUSION}

By considering this case report, patients with HF associated with preserved LV systolic function such as in restrictive cardiomyopathy, constrictive pericarditis, amyloid heart disease, and acute cor pulmonale may be particularly susceptible to the toxicity of digoxin because of the various drug-drug interactions mentioned above. In ICM, the inotropic effects of digoxin may worsen the outflow obstruction. Therapy with digoxin should be considered and administered cautiously in such patients.

Elevated INR to more than 6.0 and bleeding complications occurred after warfarin was added to the patient's regimen. The PT and INR are used to monitor the effectiveness of the anticoagulant warfarin. HF due to ischaemic or dilated cardiomyopathy is associated with a significant increase in the risk of thromboembolism. Unless there are contraindications, anticoagulant treatment is mandatory for patients with HF and atrial fibrillation. HF is an independent risk factor for over-anticoagulation.

Therefore, patients with HF should be closely monitored to prevent potential bleeding complications. And thus, the anticoagulants must be adjusted accordingly. For patients treated with warfarin, healthcare professionals should play a key role in monitoring possible interactions with other drugs, foods, herbs, and dietary supplements.

$\mathrm{HF}$ is one of the most damaging clinical outcomes for ICM patients. Heart transplantation is still the main method of long-term treatment, which can convey the protective effect of preventing heart death and improving the survival rate in the later stages of cardiac ICM. ICM causes an imbalance between myocardial oxygen demand and supply, leading to myocyte loss, myocardial scarring, and ventricular failure. 
The prevalence of ICM is not completely understood, mainly because of the lack of a standardised and universally acceptable terminology. Clinical management methods are lifestyle modifications, medical therapy, device therapy (ICD or cardiac resynchronisation therapy), revascularisation, and cardiac transplantation.

ICM is a well-recognised disease entity; however, the current classification systems of cardiomyopathy exclude cardiomyopathies secondary to ischaemia. Moreover, research on ICM is extensively fragmented, which has undermined both research consensus and comprehensive understanding.

Therefore, the purpose of the present review is to accumulate research on ICM to produce a comprehensive understanding of its clinical status, diagnosis, clinical management, and ultimately improve survival.

\section{References}

1. National Heart, Lung, and Blood Institute (NHLBI). Cardiomyopathy. 2016. Available at: https://www.nhlbi. nih.gov/health/cardiomyopathy. Last accessed: 10 November 2017.

2. GBD 2015 Mortality and Causes of Death Collaborators. Global, regional, and national life expectancy, allcause mortality, and cause-specific mortality for 249 causes of death, 1980-2015: a systematic analysis for the Global Burden of Disease Study 2015. Lancet. 2016;388(10053):14591544.

3. Practical Cardiovascular Pathology. Lippincott Williams \& Wilkins. 2010. p. 148. ISBN 9781605478418. Archived from the original on 14 September 2016.

4. GBD 2013 Mortality and Causes of Death Collaborators. Global, regional, and national age-sex specific allcause and cause-specific mortality for 240 causes of death, 1990-2013: a systematic analysis for the Global Burden of Disease Study 2013. Lancet. 2015;385(9963):117-71.

5. Weintraub RG et al. Dilated cardiomyopathy. Lancet. 2017;390(10092):400-14.

6. Ferri FF, Ferri's Clinical Advisor 2018 E-Book: 5 Books in 1 (2017) 1st $^{\text {st }}$ edition, Philadelphia: Elsevier, p.244

7. Martino TA et al. Viral infection and the pathogenesis of dilated cardiomyopathy. Circ Res. 1994;74(2):182-8.

8. Kumar $\vee$ et al. (eds.), Robbins Basic Pathology (2007) $8^{\text {th }}$ edition, Philadelphia: Saunders.

9. Pieske B. Reverse remodeling in heart failure - fact or fiction? Eur Heart J Suppl. 2004;6:D66-78.

10. Gensini GF, Rostagno C [Anticoagulant therapy in patients with dilated cardiomyopathy]. Ann Ital Med Int. 1998;13(4):227-32. (In Italian).

11. Abramson SV et al. Pulmonary hypertension predicts mortality and morbidity in patients with dilated cardiomyopathy. Ann Intern Med. 1992;116(11):888-95.

12. Felker GM et al. Underlying causes and long-term survival in patients with initially unexplained cardiomyopathy. N Engl J Med. 2000;342(15):1077-84.

13. Faber L, Lamp B. Mitral valve regurgitation and left ventricular systolic dysfunction: corrective surgery or cardiac resynchronization therapy? Herzschrittmacherther Elektrophysiol. 2008;19:52-9.

14. Yiu SF et al. Determinants of the degree of functional mitral regurgitation in patients with systolic left ventricular dysfunction: a quantitative clinical study. Circulation. 2000;102(12):1400-6.

15. Trichon $\mathrm{BH}$ et al. Relation of frequency and severity of mitral regurgitation to survival among patients with left ventricular systolic dysfunction and heart failure. Am J Cardiol. 2003;91(5):538-43.

16. Nath $\mathrm{J}$ et al. Impact of tricuspid regurgitation on long-term survival. J Am Coll Cardiol. 2004;43(3):405-9.

17. Mutlak D et al. Echocardiographybased spectrum of severe tricuspid regurgitation: the frequency of apparently idiopathic tricuspid regurgitation. J Am Soc Echocardiogr. 2007;20(4):405-8.

18. Garg $S$ et al. Association of concentric left ventricular hypertrophy with subsequent change in left ventricular end-diastolic volume: the Dallas Heart Study. Circ Heart Fail. 2017;10(8):e003959.

19. lida K et al. Pathophysiologic significance of left ventricular hypertrophy in dilated cardiomyopathy. Clin Cardiol. 1996;19(9):704-8.

20. Carabello BA, Paulus WJ. Aortic stenosis. Lancet. 2009;373(9667):956-66.

21. Korn D et al. Massive calcification of the mitral annulus. A clinicopathological study of fourteen cases. N Engl J Med. 1962;267:900-9.

22. Nestico PF et al. Mitral annular calcification: clinical, pathophysiology, and echocardiographic review. Am Heart J. 1984;107:989-96.

23. Barasch $\mathrm{E}$ et al. Clinical significance of calcification of the fibrous skeleton of the heart and aortosclerosis in community dwelling elderly. The Cardiovascular Health Study (CHS). Am Heart J. 2006;151(1):39-47.

24. Movahed MR et al. Mitral annulus calcification is associated with valvular and cardiac structural abnormalities. Cardiovasc UItrasound. 2007;5:14

25. Nikolaidou $\mathrm{T}$ et al. Outcomes related to first-degree atrioventricular block and therapeutic implications in patients with heart failure. JACC Clin Electrophysiol. 2016;2(2):181-92.

26. Duchnowski $P$ et al. High sensitivity troponin $\mathrm{T}$ as a prognostic marker in patients undergoing aortic valve replacement. Pol Arch Intern Med. 2017;127(9):628-30.

27. Duchnowski $P$ et al. High-sensitivity troponin T predicts postoperative cardiogenic shock requiring mechanical circulatory support in patients with valve disease. Shock 2020;53(2):175-8.

28. U.S. Food and Drug Administration (FDA). LANOXIN (digoxin) tablets, for oral use. 2016. Available at: https:// www.accessdata.fda.gov/drugsatfda_ docs/label/2016/020405s013lbl.pdf. Last accessed: 26 August 2021.

29. U.S. Food and Drug Administration (FDA). Micardis (telmisartan) tablets. 2011. Available at: https://www. accessdata.fda.gov/drugsatfda_docs/ label/2011/020850s032lbl.pdf. Last accessed: 26 August 2021.

30. U.S. Food and Drug Administration (FDA). Lopressor ${ }^{\circledR}$ metoprolol tartrate injection, USP 2013. Available at: https://www.accessdata. fda.gov/drugsatfda_docs/ label/2013/018704s026lbl.pdf. Last accessed: 26 July 2013. 
31. Lilly LS (ed.), Pathophysiology of heart disease: a collaborative project of medical students and faculty (2011) $5^{\text {th }}$ edition, Baltimore: Lippincott Williams \& Wilkins.

32. Langsjoen $\mathrm{PH}$ et al. A six-year clinical study of therapy of cardiomyopathy with coenzyme Q10. Int J Tissue
React. 1990:12(3):169-71.

33. Folkers $\mathrm{K}$ et al. Therapy with coenzyme Q10 of patients in heart failure who are eligible or ineligible for a transplant. Biochem Biophys Res Commun. 1992;182(1):247-53.

34. Baggio E et al. Italian multicenter study on the safety and efficacy of coenzyme Q10 as adjunctive therapy in heart failure. CoQ10 Drug Surveillance Investigators. Mol Aspects Med. 1994;15:s287-94.

35. Rabow MW et al. (eds.), Current medical diagnosis and treatment 2017 (2016) 56 $6^{\text {th }}$ edition, New York: McGraw-Hill Medical. 